\title{
Very High-Energy Gamma Ray Astronomy
}

\author{
Ullrich Schwanke \\ Institute of Physics \\ Humboldt University Berlin \\ Newtonstrasse 14 \\ 12489 Berlin, GERMANY
}

\section{Overview}

Very high-energy (VHE) gamma ray astronomy explores the non-thermal Universe at energies greater than $\sim 100 \mathrm{GeV}$. This contribution to the XXVIII Physics in Collision Conference describes the status of the field and gives examples (and references) for advances that have been obtained since the last presentation in this conference series [1].

\section{Experimental Techniques and Experiments}

The fluxes of VHE photons are so small that their detection requires collection areas far bigger $\left(\mathcal{O}\left(10^{4}-10^{5} \mathrm{~m}^{2}\right)\right)$ than those of typical satellite-borne experiments $\left(\mathcal{O}\left(1 \mathrm{~m}^{2}\right)\right)$. The best flux sensitivity is obtained by Imaging Atmposheric Cherenkov Telescopes (IACTs) which detect the Cherenkov light emitted by the showers that are initiated by gamma-rays and hadrons in the atmosphere. The Cherenkov light is imaged onto a camera and can be used to reconstruct the direction, energy and type (photon or hadron) of the incoming particles in a typical $4^{\circ}$-diameter field of view $(0.004 \mathrm{sr})$. Often, the shower is observed by more than one telescope (stereoscopic observation) resulting in a better determination of shower parameters and improved

background rejection. Arrival directions are reconstructed with a typical accuray of $0.05-0.10^{\circ}$ (depending on energy) while the energy estimate has an error of $\sim 20 \%$.

The major IACT systems operating at present are CANGAROO ([2], Australia), H.E.S.S. ([3], Namibia), MAGIC ([4], Canary Islands), and VERITAS ([5], Arizona). Their overall duty cycle is $\sim 15 \%$ since observations require cloudless nights with no or little moonshine. A source similar to the Crab Nebula (the standard candle of VHE astronomy) is detected by H.E.S.S. in $30 \mathrm{~s}$. In a typical exposure of 25 hours at a zenith angle of $20^{\circ}$, a source with $1 \%$ of the Crab Nebula flux is detected at the $5 \sigma$ level. 
At higher energies (few $\mathrm{TeV}$ ), non-imaging air shower detectors identify showers initiated by photons using different experimental techniques. The so called Water Cherenkov Technique has been pioneered by MILAGRO ([6], New Mexico), while other installations (ARGO and Tibet AS Gamma ([7, 8], Tibet)) use a mixture of resistive plate chambers and scintillators. Unlike the IACTs, the non-imaging experiments cover only the northern hemisphere and have a limited angular resolution $\left(\sim 1^{\circ}\right)$. Despite their good duty cycle $(\sim 90 \%)$ and large field of view $(\sim 2 \mathrm{sr})$ the obtained sensitivity is far lower than those of IACTs; a detection of the Crab Nebula requires an exposure of ( $\sim 3$ months $)$.

\section{Source Counts and Source Types}

In the past 5 years, the number of known VHE gamma ray sources has increased considerably. The TeV catalogue [9] lists now $\sim 70$ sources, $\sim 50$ of which are galactic. Roughly two thirds of all sources were discovered by the H.E.S.S. array of Cherenkov telescopes that performed a systematic scan of the Galactic plane (see Section 4).

Among the galactic sources, pulsar wind nebulae (see [10] for a review) are the most numerous ( $\sim 10$ detections), followed by supernova remnants (see Section 6 ). Also detected were $4 \mathrm{TeV}$ binaries $[11,12,13,14,15]$, at least one stellar association [16], and there a $\sim 3$ examples were VHE gamma rays were found coincident with molecular clouds. Searches for VHE gamma rays from dwarf galaxies orbiting the Milky Way remained unsuccessful and were used to establish limits $[17,18]$ in the context of dark matter studies. The Galactic Centre source [19] was recently shown to be spatially separated from a nearby supernova remnant, excluding this explanation for the observed VHE radiation.

The $\sim 20$ extra-galactic sources are all of the blazar type (see Section 7 ) with the exeption of the giant radio galaxy M87. Searches for VHE radiation from other extragalactic objects (starburst galaxies and galaxy clusters) have yielded upper limits, indicating that these systems are too faint and/or too far away to be visible for current instruments. Observations of gamma ray burst (following triggers from satellites) were conducted either directly or in follow-up mode. MAGIC managed to observe a GRB only $40 \mathrm{~s}$ after its onset, and H.E.S.S. was lucky enough to serendipitiously catch a GRB at the edge of its field of view, but searches for an excess of VHE photons remained unsuccessful [20,21, 22].

\section{The Milky Way in VHE Gamma Rays}

The southern location of the H.E.S.S. experiment and its large field of view $\left(5^{\circ}\right.$ diameter) make it ideally suited for a systematic scan for VHE gamma ray sources in 
the inner Galaxy. Such a scan was performed in 2004 and 2005 between $\pm 30^{\circ}$ in galactic longitude and $\pm 2^{\circ}$ in latitude and led to the discovery of 8 sources[23]. The scan was later extended to $\pm 3^{\circ}$ in latitude, and to $+60^{\circ}$ and $-85^{\circ}$ in longitude, and increased the number of found sources to $>14$ [24]. The typical sensitivity obtained by H.E.S.S. in the scan is few per cent of the Crab. A variety of sources -most of them extended- was found and a good fraction of the sources cannot be plausibly associated with sources known from other wavelength bands. Multi-wavelength observations are underway to identify counterparts.

The northern Galaxy was observed by MILAGRO in the years 2000-2007 and the total data set was recently published [25]. The sensitivity level reached by MILAGRO is a factor of 10 worse than that of H.E.S.S., but the observations extend to $\pm 10^{\circ}$ in latitude (mainly limited by the usable zenith angle band) and cover $+30^{\circ}$ to $+220^{\circ}$ $\left(=-140^{\circ}\right)$ in longitude. The analysis of the MILAGRO data set (6.5 years of livetime) provided a clear detection of the Crab Nebula and the discovery of seven sources three of which are significant after accounting for trials. Two of the sources are found in the Cygnus region where HEGRA and MAGIC also reported TeV excesses[26, 27], but MILAGRO's coarse directional resolution makes a detailed comparison difficult. The third source was found in the overlap window of the MILAGRO and H.E.S.S. observations $\left(+30^{\circ}\right.$ to $+60^{\circ}$ in longitude). This source (MGRO 1908+06), was recently confirmed by H.E.S.S. [28]. A 15 h exposure revealed a new source (HESS J1908+63) at a significance of $5.7 \sigma$ coincident with the reported MILAGRO detection. The energy spectrum of the fairly strong source (14\% Crab) was measured between 0.4 and $25 \mathrm{TeV}$ and appears hard (spectral index $\Gamma=2.05 \pm 0.06$ ), and there is evidence for an association with a SNR and/or molecular clouds. While MILAGRO was unable to derive a spectrum of the source, the MILAGRO excess converted into a differential flux measurement at $\sim 20 \mathrm{TeV}$ aligns well with the H.E.S.S. spectrum. These MILAGRO and H.E.S.S. results demonstrate nicely that a consistent picture of our Galaxy at $\mathrm{TeV}$ energies is emerging.

\section{The Crab Nebula and its Pulsar}

The numerous detections in the VHE band have significantly improved our understanding of the structure and evolution of pulsar wind nebulae (see [29] for a recent review). We want concentrate here on the well-known Crab Nebula where measurements with the MAGIC telescope have allowed the determination of the spectrum down to $60 \mathrm{GeV}$ and, for the first time in the history of ground-based gamma ray astronomy, the detection of pulsed emission from a pulsar at around $\sim 25 \mathrm{GeV}$.

The Crab Nebula is the remnant of a supernova explosion from 1054 AD. Its diameter is just a few light years, so it appears - at a distance of $\sim 2 \mathrm{kpc}-$ point-like for IACTs. Its steady spectrum has been measured over many decades in energy and 
is understood in terms of a synchrotron self-Compton (SSC) scenario where electrons accelerated at the pulsar wind termination shock generate a synchrotron spectrum ranging from radio frequencies to $\mathrm{GeV}$ energies. The inverse-Compton upscattering of the very same synchrotron photons by the electron population results in a second hump at higher energies than the synchrotron part of the nebula's spectrum. Earlier observations with IACTs detected the falling edge of the inverse Compton hump, but only recently the low energy threshold of the MAGIC telescope has provided evidence for the actual existence of an inverse Compton peak [30]. The MAGIC measurements down to $60 \mathrm{GeV}$ are well described by inverse Compton emission and place the inverse Compton peak at $\sim 77 \mathrm{GeV}$.

The pulsar powering the Crab Nebula (PSR B0531+21) has a period of $33 \mathrm{~ms}$ and features the largest energy output $\left(5.1 \cdot 10^{38} \mathrm{erg} \mathrm{s}^{-1}\right)$ among all known galactic pulsars. Pulsed emission at the pulsar period has been detected from the radio band up to $\sim 5 \mathrm{GeV}$ and appears in two peaks in the phasogram. MAGIC found a first hint for pulsed emission $(2.9 \sigma)$ in $16 \mathrm{~h}$ of standard data-taking. Data-taking with a dedicated pulsar trigger electronics between October 2007 and February 2008 resulted in a $22.3 \mathrm{~h}$ data set at an energy threshold that could be reduced from $50-60 \mathrm{GeV}$ to $25 \mathrm{GeV}$. In an analysis of the data set, $\sim 8500$ photons from pulsed emission could be identified $(6.4 \sigma)$. An analysis of the energy spectrum of the pulsed photons shows a cutoff around $16-21 \mathrm{GeV}$ which indicates that the emission region must be placed far out in the magnetosphere which disfavours in particular the polar cap emission scenario[31, 32, 33].

The detection of pulsed emission from the Crab Nebula by MAGIC is a very significant result since it demonstrates for the very first time that low-threshold IACTs can bridge the gap to gamma-ray satellites and provide important and novel input to deepen our understanding of the emission processes in the magnetosphere of pulsars.

\section{Supernova Remnants as Cosmic Ray Sources}

Shell-type supernova remnants (SNR) are suspected acceleration sites for hadronic cosmic rays with energies up to the knee. The presence of high-energy electrons had been derived earlier from the detection of non-thermal synchrotron emission at X-ray energies, but the presence of accelerated hadrons in the SNR shock fronts remained hard to prove. H.E.S.S. observations of shell-type SNRs identified the shells of two objects (RX J1713.7-3946 [34, 35, 36] and RX J0852.0-4622[37, 38]) as VHE emitters, and there is a third SNR (RCW 86[39]) where the shell morphology is not as clear. The detection of VHE photons at several $10 \mathrm{TeV}$ and a strong apparent correlation of the VHE emission with X-ray observations are striking evidence that the SNR shells accelerate particles to energies of $\mathcal{O}(100 \mathrm{TeV})$. A modelling of the multi-wavelength spectra of RX J1713.7-3946 and RX J0852.0-4622 in an electronic 
scenario (where the VHE emission is attributed to inverse Compton scattering of electrons on low-energy photon fields) results in rather low magnetic fields (about the size of the $B$ field in the interstellar medium) which is at odds with the diffusive shock acceleration mechanism and probably also with the thin filaments observed at X-ray energies. While hadronic models (VHE photons from $\pi^{0}$ decays generated in the interaction of accelerated with the interstellar medium) can describe the VHE spectra fairly well, there is still lack of evidence that protons are really accelerated at the required efficiency and that the generated hadron spectra extend up to the knee.

In this situation (and facing the small number of available young SNRs) observations at VHE energies have also been extended to older SNRs and it has been tried to identify associations of SNRs and molecular clouds. Only relatively young (up to the Sedov phase) SNRs can shock-accelerate particles to PeV energies, but somewhat older SNRs could be interesting since electrons suffer from stronger energy losses while proton populations might survive and still generate observable signals. Another option is the illumination of nearby dense molecular clouds by high-energy protons that escape the acceleration zone, diffuse out and still interact in the dense molecular cloud while efficient particle accelaration might have ceased in the SNR [40].

Indeed, observations by MAGIC (e.g. IC 444[41]) and H.E.S.S. (e.g. W28[42], HESS J1745-303[43], CTB 37B[44]) have now identified a number of VHE gamma ray sources that appear associated with the shells of SNRs (but are unlikely pulsar wind nebulae), or with molecular clouds whose extent and distance can be inferred from observations in other wavebands (in particular CO observations). A spatial correlation of the VHE sources with molecular clouds and/or regions of maser emissions is observed and is suggestive of a hadronic and SNR-related origin of the VHE emission. Unfortunately, substantial uncertainties on important parameters of SNRs (age, distance, matter density) and clouds (exact kinematic distance, density) do often not allow the firm exclusion of alternative explanations of the observed VHE sources. It appears not unlikely that better instruments with improved sensitivity (in order to boost the source statistics) and wider energy coverage (for a longer lever arm in spectra) will be required to unambigiously identify SNRs as cosmic ray sources.

\section{Active Galactic Nuclei}

New Active Galactic Nuclei (AGN) that emit VHE gamma rays are being discovered by IACTs at a rate of $\mathcal{O}(5)$ per year. With one exception $(\mathrm{M} 87[45,46])$, all detected AGN are of the blazar type where the jet is directed toward the observer. The improved source statistics and the detection of spectacular flares (e.g. from PKS 2155-30 where fluxes at the level of $\sim 15$ Crab, i.e. 100 times the low-state flux [47], were observed) with short rise times $(\sim 100 \mathrm{~s})$ resulted in important constraints on the 
size of the emission region and provided input for the modelling of the AGN emission. At the same time, the observation of AGN at substantial redshifts $(z>0.1)$ further constrains the amount extra-galactic background light (EGBL) which attenuates the intrinsic AGN spectra by photon-photon pair production over cosmological distances. In particular, a new AGN discovered by H.E.S.S. at $z=0.14$ (1ES 0229+200[48]) can be used to show that the Universe is more transparent in the $2-20 \mu \mathrm{m}$ window. If one assumes that the intrinsic spectrum cannot be arbitrarily hard the new data disfavour EGBL models with a high mid infrared flux. It is too early to speak of high-precision cosmology with AGN here (especially given the fact that our understanding of the intrinsic AGN spectra is still far from complete), but it is also clear that the derived constraints are challenging models and direct EGBL measurements. Only recently, the detection of two AGN at redshifts of $z=0.212$ (1ES 1011+496[49]) and $z=0.538$ (3C279[50]) by MAGIC doubled the range of redshifts where AGN emission can be studied, which should make the EGBL limits even more constraining.

\section{Summary and Outlook}

The current IACTs (notably H.E.S.S., MAGIC and VERITAS) explore the sky with regular observations between $60-100 \mathrm{GeV}$ and some $10 \mathrm{TeV}$ and reach a sensitivity of few per cent Crab. The number of known sources is growing and will soon approach $\sim$ 100. Scan-type observations of H.E.S.S. in the south and the publication of the complete MILAGRO data set (albeit at a much higher threshold than that of IACTs) for the north provide a first picture of the Milky Way at VHE energies. IACTs collect evidence for SNRs as cosmic ray sources and address fundamental physics question ranging from electrodynamics (pulsar magnetospheres) to cosmology. At the time of writing, the existing IACTs are being upgraded to obtain lower thresholds (H.E.S.S. adds a $28 \mathrm{~m}$ diameter telescope with an anticipated trigger threshold of $\sim 25 \mathrm{GeV}$ ) and better background rejection by stereoscopic observations (MAGIC adds a second $17 \mathrm{~m}$ diameter telescope). For the next few years, a lot can be expected from these upgrades, the overlap with the recently launched FERMI[51] satellite and studies of the anisotropies reported by AUGER[52]. In the next decade, VHE gamma ray astronomy might enter a new era with the design and construction effort for the Cherenkov Telescope Array (CTA[53]) which is expected to provide an order of magnitude better sensitivity and much wider energy coverage.

\section{References}

[1] M. Teshima, Talk at the XXVI Physics in Collision conference (2006).

[2] The CANGAROO website (http://icrhp9.icrr.u-tokyo.ac.jp). 
[3] The H.E.S.S. website (http://www.mpi-hd.mpg.de/hfm/HESS).

[4] The MAGIC telescope website (http://wwwmagic.mppmu.mpg.de).

[5] The VERITAS website (http://veritas.sao.arizona.edu).

[6] The MILAGRO website (http://www.lanl.gov/milagro/index.shtml).

[7] The ARGO website (http://argo.na.infn.it).

[8] The Tibet AS Gamma Experiment website (http://www.icrr.utokyo.ac.jp/em/index.html).

[9] The TeV Catalogue website (http://www.tevcat.ucicago.edu).

[10] Gaensler, B. M., \& Slane, P. O., Ann. Rev. Astron. Astrophys., 44, 17 (2006).

[11] F. Aharonian et al. [The H.E.S.S. Collaboration], A\&A 460, 743-749 (2006).

[12] F. Aharonian et al. [The H.E.S.S. Collaboration], A\&A 442, 1-10 (2005).

[13] J. Albert et al., Science 312, 1771 (2006).

[14] J. Albert et al., Astrophys. J. 684, 1351 (2008).

[15] J. Albert et al., Astrophys. J. Lett. 665, L51 (2007).

[16] F. Aharonian et al. [The H.E.S.S. Collaboration], A\&A 467, 1075-1080 (2007).

[17] F. Aharonian et al. [The H.E.S.S. Collaboration], Astroparticle Physics 29, 55-62 (2008).

[18] J. Albert et al., Astrophys. J. 679, 428 (2008).

[19] H.E.S.S. collaboration, Localising the H.E.S.S. Galactic Center point source, Proc. 30th International Cosmic Ray Conference, Merida, (2007).

[20] F. Aharonian et al. [The H.E.S.S. Collaboration], Accepted for publication in Astrophys. J.

[21] J. Albert et al., ApJ Letters 641, L9 (2006).

[22] J. Albert et al., Astrophys. J. 667, 358 (2007).

[23] F. Aharonian et al. [The H.E.S.S. Collaboration], Science 307, 1938-1942 (2005).

[24] F. Aharonian et al. [The H.E.S.S. Collaboration], Astrophys. Journal 636, 777797 (2006). 
[25] A. A. Abdo et al., Astrophysical Journal 664, L91 (2007).

[26] F. Aharonian et al. [The HEGRA Collaboration], A\&A 393, L37 (2002).

[27] J. Albert et al., Astrophys. J. 675, L25 (2008).

[28] H.E.S.S. collaboration, H.E.S.S. Galactic Plane Survey unveils a Milagro Hotspot, Proc. 30th International Cosmic Ray Conference, Merida, (2007).

[29] O.C. de Jager, A. Djannati-Ataï, Springer Lecture Notes on Neutron Stars and Pulsars: 40 years after their discovery (2008).

[30] The MAGIC collaboration: E. Aliu, et al (http://arxiv.org/abs/0809.2998).

[31] Baring, M. G., Adv. Space Res., 33, 552-560 (2004).

[32] Thompson, D.J., Bertsch, D.L. \& O'Neal, R.H.,Astrophys. J. Suppl. Ser., 157, 324-334 (2005).

[33] Ruderman, M.A., Sutherland, P.G., Theory of Pulsars- Polar caps, sparks, and coherent microwave radiation, Astrophys. J., 196, 51-72 (1975).

[34] F. Aharonian et al. [The H.E.S.S. Collaboration], Nature 432, 75 (2005).

[35] F. Aharonian et al. [The H.E.S.S. Collaboration], A\&A 449, 223-242 (2006).

[36] F. Aharonian et al. [The H.E.S.S. Collaboration], A\&A 464, 235-243 (2007).

[37] F. Aharonian et al. [The H.E.S.S. Collaboration], A\&A 437, L7 (2005).

[38] F. Aharonian et al. [The H.E.S.S. Collaboration], Astrophys. Journal 661, 236249 (2007).

[39] H.E.S.S. collaboration, H.E.S.S. observations of the supernova remnant $R C W$ 86, Proc. 30th International Cosmic Ray Conference, Merida, (2007).

[40] S. Gabici, F.A. Aharonian and P. Blasi, Astroph. Space Sci. 309, 365-371 (2007).

[41] J. Albert et al., ApJ Lett. 664, L87 (2007).

[42] F. Aharonian et al. [The H.E.S.S. Collaboration], A\&A 481, 401-410 (2008).

[43] F. Aharonian et al. [The H.E.S.S. Collaboration], A\&A 483, 509-517 (2008).

[44] F. Aharonian et al. [The H.E.S.S. Collaboration], A\&A 486, 829-836 (2008).

[45] F. Aharonian et al. [The H.E.S.S. Collaboration], Science 314, 1424-1427 (2006). 
[46] J. Albert et al., Astrophys. J. Lett. 685, L23 (2008).

[47] F. Aharonian et al. [The H.E.S.S. Collaboration], Astrophys. Journal Lett. 664, L71-L74 (2007).

[48] F. Aharonian et al. [The H.E.S.S. Collaboration], A\&A 475, L9-L13 (2007).

[49] J. Albert et al., Astrophys. J. Lett. 667, L21 (2007).

[50] MAGIC Collaboration, Science, Vol. 320, 1752 (2008).

[51] The Fermi telescope website (http://www-glast.stanford.edu).

[52] The Pierre Auger Collaboration et al., Science Vol. 318, 938-943 (2007).

[53] The CTA website (http://www.cta-observatory.org). 